\title{
The Role of Predictors Parameters in the Management Policy and Outcome of Congenital Diaphragmatic Hernia with the Lack of Resources in Single Pediatric Surgery Unit
}

\author{
Mohammed Joudi Aboud \\ Pediatric Surgery Unit, the Maternity and Child Teaching Hospital, Al Qadisiya, Iraq \\ Email address: \\ mohammedabud@yahoo.com
}

To cite this article:

Mohammed Joudi Aboud. The Role of Predictors Parameters in the Management Policy and Outcome of Congenital Diaphragmatic Hernia with the Lack of Resources in Single Pediatric Surgery Unit. International Journal of Biomedical Engineering and Clinical Science.

Vol. 3, No. 6, 2017, pp. 110-118. doi: 10.11648/j.ijbecs.20170306.17

Received: April 3, 2017; Accepted: April 21, 2017; Published: January 8, 2018

\begin{abstract}
Background: The objective of this study is to have the interest to know how far the present study underscores the improved survival rate for patients with $\mathrm{CDH}$ and risk factors affecting the outcome with the lack of many essential facilities. Our inquiries: shall we proceed without ECMO and related resources! Patients and Methods: The records of patients admitted with CDH to the pediatric surgery unit at the Maternity and Children Teaching Hospital Al Qadisiya, Iraq, from January 1, 2005, to the end of December 2014 were retrospectively reviewed. Reports obtained about operative findings, respiratory complications, although attempts were made to confirm a suspected diagnosis of pulmonary hypertension using echocardiography. Despite that, the permissive hypercapnia and ECMO were not practiced during the study period (these facilities still unavailable in our unit), we considering that the studied patients suspected to have such facilities according to ECMO protocol. All presentation factors with some of the predictive parameters are studied and taken as background to analyze our study. Statistical analyses performed using the Statistical Package for Social Sciences (SPSS) version 20 software and Excel 2016. Independent t-test used for analysis of normally distributed continuous data. A p-value of $<.0001$ considered statistically significant. Results: Thirty-one patients $(26$ neonates) were managed at our unit with an overall survival 19 of the total patients $73.07 \%$ and 14 (53.84\%) of the neonates. Male presented 18 (69.23\%) out of the total. Birth weight in grams (range) 3100 (2100 to 4390). Apgar (mean) at 5 min. after delivery (range) $6.2 \pm 2.1$. At the level of Prenatal factors, chest to head circumference ratio $<0.80$ presented as the most important risk factor (Odd ratio 27.50, 95\% confidence intervals (CI) upper 289.13 and $p$-value $<.0001$ ). At birth factors, associated major anomalies (Odd ratio 3.50, 95\% CI upper 17.89 and p-value <.0001) formed risk parameter. Overall, both favorable and unfavorable factors had significant correlation and predictive effects on both survival and mortality groups respectively. Conclusion: A composite of prognostic and predictive parameters, however, organize our workup and can magnify our management future policy despite that, these prognostic indicators measure different aspects of $\mathrm{CDH}$.
\end{abstract}

Keywords: Predictive Parameters, Management Policy, Congenital Diaphragmatic Hernia

\section{Introduction}

Congenital diaphragmatic hernia $(\mathrm{CDH})$ is a disorder characterized by failure of the pleural and peritoneal canal to close approximately at the 7-8 week of gestation. This leads to the evisceration of the abdominal contents into the thoracic cavity resulting in pulmonary hypoplasia due to compression of the developing lungs by the viscera. Hernias through the Foramen of Bochdalek (a posterolateral aspect of the diaphragm) account for approximately $80 \%$ of hernias, and hernias on the left side are five times more common than on the right side. With the wide use of obstetric ultrasound, prenatal diagnosis of these hernias is common. Neonates usually present with asphyxia, respiratory distress, cyanosis and scaphoid abdomen. Those who develop respiratory failure within the first six hours of life have the highest mortality. This mortality rate has not changed significantly over the last decade, despite changes in ventilation management, the use of vasoactive and inotropic medications and delaying surgical repair attempting to minimize any postoperative reduction in 
lung compliance [1-4]. Despite the advances in neonatal intensive care and ventilation facilities, the mortality remains frustratingly high. Various predictive parameters and prognostic indicators have been suggested for the survival of babies with $\mathrm{CDH}$. These include birth weight, APGAR scores, the age of presentation, associated congenital anomaly and ventilation scores like ventilation index, $\mathrm{pCO}_{2}, \mathrm{pO}_{2}$, and oxygenation index. Other indicators are complications like pneumothorax, persistent pulmonary hypertension (PPHN) and types of therapies used like hyperventilation, drugs and extracorporeal membrane oxygenation (ECMO) [5].

Congenital diaphragmatic hernia $(\mathrm{CDH})$, although with rare incidence, about 1 per 2,000-5,000 births, is associated with high mortality. Specialized centers with extracorporeal membrane oxygenation (ECMO) facilities reported promising survival rates percentage. Current management strategies consist of preoperative stabilization and delayed repair [6]. Various other treatment strategies initiated by different centers to improve outcome [7, 8]. Prenatal therapies such as prenatal surgery or temporary occlusion of the fetal trachea were not enrolled and we have no such experience in this field of surgery. lack of resources magnifies the challenge of management and surgery as our unit does not offer ECMO support (not available in our country) and the optimal management of our patients delivered with such pathology carry many challenges. We have the interest to know how far the present study underscores the improved survival rate for patients with $\mathrm{CDH}$ and risk factors affecting the outcome with the lack of many essential facilities. Our inquiries: shall we proceed without ECMO and related resources!

\section{Patients and Methods}

The records of patients admitted with $\mathrm{CDH}$ to the pediatric surgery unit at the Maternity and Children Teaching Hospital Al Qadisiya, Iraq, from January 1, 2005, to the end of December 2014 were retrospectively reviewed. The data submitted included gestational age, sex, birth weight, Apgar score at one and five minutes, antenatal diagnosis, mode of delivery, inborn or outborn and age of onset of respiratory symptoms. Reports obtained about operative findings (the side of a hernia and the abdominal contents in the chest), respiratory complications (PPHN, air leak syndrome), although attempts were made to confirm a suspected diagnosis of pulmonary hypertension using echocardiography. Associated congenital anomalies and survival to discharge were also reviewed. In our policy, a cut-off postnatal age of 24 hours was selected in order to include infants from referral hospitals or primary health care centers in the region as well as to minimize the effects of postnatal physiological changes and interventions on respiratory compliance. All with diaphragmatic eventration were excluded from this study. Data about initial preoperative preductal arterial oxygen tension $\left(\mathrm{PaO}_{2}\right)$, and age at surgical repair, also submitted.

Despite that, the permissive hypercapnia and ECMO were not practiced during the study period (these facilities still unavailable in our unit), we considering that the studied patients suspected to have such facilities according to ECMO protocol: Gestational age $\geq 34$ weeks, Weight $\geq 1.8 \mathrm{~kg}$, Reversible disease, Failure of maximal medical management and Predicted mortality $\geq 80 \%$ by historical criteria.

Table 1. Proposed predictors of outcome submitted to this study.

\begin{tabular}{|c|c|c|}
\hline & Proposed favorable outcome & Proposed unfavorable outcome \\
\hline \multirow{4}{*}{ Prenatal factors } & lung-to-head ratio $>1.0$ & lung-to-head ratio $>1.0$ \\
\hline & chest-to-abdomen circumference ratio $>0.95$ & chest-to-abdomen circumference ratio $<0.95$ \\
\hline & chest-to-head circumference ratio $>0.80$ & chest-to-head circumference ratio $<0.80$ \\
\hline & No liver herniation & liver herniation \\
\hline \multirow{4}{*}{ Factors at birth } & No associated anomalies & associated anomalies \\
\hline & Birth weight $>3000 \mathrm{~g}$ & Birth weight $<3000 \mathrm{~g}$ \\
\hline & Delivery at hospital obstetric center & Delivery at community (midwife) \\
\hline & High -5 min. apgar & Low -5 min. apgar \\
\hline \multirow[t]{3}{*}{ Early postnatal patient factors } & Preductal $\mathrm{SaO}_{2}>90 \%$ in 1 st 24 hour & Preductal $\mathrm{SaO}_{2}<90 \%$ in 1 st 24 hour \\
\hline & Spontaneous ventilation & $(+)$ Pressure ventilation/muscle paralysis \\
\hline & No pneumothorax & Pneumothorax requiring a chest tube \\
\hline \multirow[t]{4}{*}{ ICU* and operative management } & Delayed surgical repair & Early surgical repair \\
\hline & Primary diaphragm Repair & Patch diaphragm Repair \\
\hline & PPHT** not documented $^{*}$ & PPHT documented \\
\hline & Oxygen index $* * *<0.4$ & Oxygen index $>0.4$ \\
\hline \multirow[t]{2}{*}{ preoperative ventilator support } & Ventilation index $* * * *<90$ & Ventilation index $>90$ \\
\hline & Post ductal $\mathrm{O}_{2}$ Saturation $>85 \%$ & Post ductal $\mathrm{O}_{2}$ Saturation $<85 \%$ \\
\hline
\end{tabular}

$*$ Intensive care unit, ** Persistent pulmonary hypertension, $* * *$ for $>4$ hours calculated $=\left[\right.$ Mean airway pressure $\times$ fraction of inspired oxygen $\left.(\text { FiO })_{2} \times 100\right] /$ post ductal $\mathrm{PaO}_{2}, * * * *$ calculated $=$ partial pressure of arterial $\mathrm{CO}_{2}\left(\mathrm{PCO}_{2}\right) \times$ [the peak airway pressure (PIP) - positive end-expiratory pressure $\left.(\mathrm{PEEP})\right] \times$ the rate of ventilation $(\mathrm{RR})] / 1000$.

The principal endpoints were hospital/operative mortality and total overall (crude) survival, (defined as the proportion of patients alive at the end of the study). Operative mortality (OM) defined as patients that died within 30 days from surgery and hospital mortality (HM) for patients that did not leave the hospital alive. All presentation factors with some of the predictive parameters [9] are studied and taken as background to analyze our study (table 1). Major emphasis pointed to those with proposed unfavorable outcome. The outcome according to the prognostic indicators were reviewed. Data translated into a computerized database. Expert statistical advice sought. Statistical analyses performed using the Statistical Package for 
Social Sciences (SPSS) version 20 software and Excel 2016. Independent t-test used for analysis of normally distributed continuous data. A p-value of $<.0001$ considered statistically significant. Our results are submitted with the mean \pm standard error of the mean for continuous data and as percentages for categorical variables.

\section{Results}

Thirty-one patients (26 neonates) were managed at our unit with an overall survival 19 of the total patients $73.07 \%$ and 14 (53.84\%) of the neonates. Five patients $(16.12 \%)$ were late presenters (26 days- 23 months), otherwise healthy and were operated on successfully without any major complications. Male presented $18(69.23 \%)$ out of the total. Birth weight in grams (range) 3100 (2100 to 4390). Apgar (mean) at 5 min. after delivery (range) 6.2 \pm 2.1 . A prenatal diagnosis of isolated $\mathrm{CDH}$ recorded in 12 neonates. The major congenital anomaly was present in $12(46.15 \%)$ of the neonates' cases, These anomalies included significant dysmorphic features, genitourinary anomalies, musculoskeletal anomalies, cardiovascular anomalies, neurologic anomalies, other gastrointestinal anomalies, chromosomal anomalies and craniofacial anomalies. Five case subjects had recognized syndromes. Gestational age for the neonates were (range) 38 (36 to 42) weeks. A hernia was on the left side in 24 cases $(77.4 \%)$, on the right side in 4 cases $(12.9 \%)$, bilateral in 2 cases $(6.4 \%)$ and central hernia in one case $(3.2 \%)$. The median length of hospital stay was 14 days (range 1-30 days). Post-operative and hospital mortality in the neonates were $3(9.6 \%)$ and $9(29 \%)$ respectively.

At the level of Prenatal factors, chest to head circumference ratio $<0.80$ presented as the most important risk factor (Odd ratio 27.50, 95\% confidence intervals (CI) upper 289.13 and p-value <.0001) (figure 1). The mean survival of the neonates with proposed favorable outcome 11.25 , SE $0.6292, \mathrm{CI}$ at $95 \% \pm 2.0007$ with the significant relation to the outcome (p-value <.0001) (table 2). A significant effect to the unfavorable outcome factors at this level (mean mortality 8.5, SE 0.5 and p-value <.0001).

At birth factors, despite the slight effect of both supposed favorable and unfavorable factors on the total outcome, associated major anomalies (Odd ratio 3.50, 95\% CI upper 17.89 and $\mathrm{P}$-value <.0001) and birth weight $<3000$ formed risk parameters (Odd ratio 29.25, 95\% CI upper 247.69 and $\mathrm{P}$-value $<.0001$ ) respectively (figure 2 ).

Pre ductal $\mathrm{SaO}_{2}<90 \%$ play an important role as a risk factor at the early post-natal level (Mean (average) of mortality 7.66, standard deviation SD 0.577 , Odd ratio 4.81, 95\% CI upper 25.79 and P-value <.0001) (figure 3).

At the intensive care and operative level, 9 of patients were kept on ventilator postoperatively (mean 2.1 days, range 1-4 days). The positive pressure ventilation/muscle paralysis and the ventilation index Ventilation index $>90$ reflected the significant risk effect of the unfavorable outcome factors (Odd ratio 4.80, 95\% CI upper 27.20 and P-value $<.0001$ ), (Odd ratio 20.00, 95\% CI upper 175.04 and P-value <.0001) respectively (figure 4). A significant correlation between favorable factors and survived patients (mean 12.6, SE 0.5099 and $\mathrm{P}$-value <.0001).

For 22 of 31 patients received preoperative ventilator support, a significant correlation between Oxygen index $>0.4$ as a predictive favorable factor on the survived patients (Odd ratio $0.02,95 \% \mathrm{CI}$ upper 0.30 and $\mathrm{P}$-value $<.0001$ ) (figure 5 ).

Although the ECMO facilities did not enroll in our unit, those patients under the categories of ECMO protocol were proceeded for surgery (figure 6). No obvious statistical significance difference between survived and not survived ( $F$ test for the significance of the difference between the variance of the two sample: $\mathrm{p}$-value 0.175407$), \mathrm{P}$ one-tailed 0.0591165 . Mean survived (observed) was 13.4 , CI $95 \%$ and $99 \%$ were \pm 6.2535 and \pm 10.3474 respectively.

Overall, both favorable and unfavorable factors had significant correlation and predictive effects on both survival and mortality groups respectively (table 3 ).

Table 2. Statistical results of the observed effects of favorable versus unfavorable factors on the survival and mortality outcome.

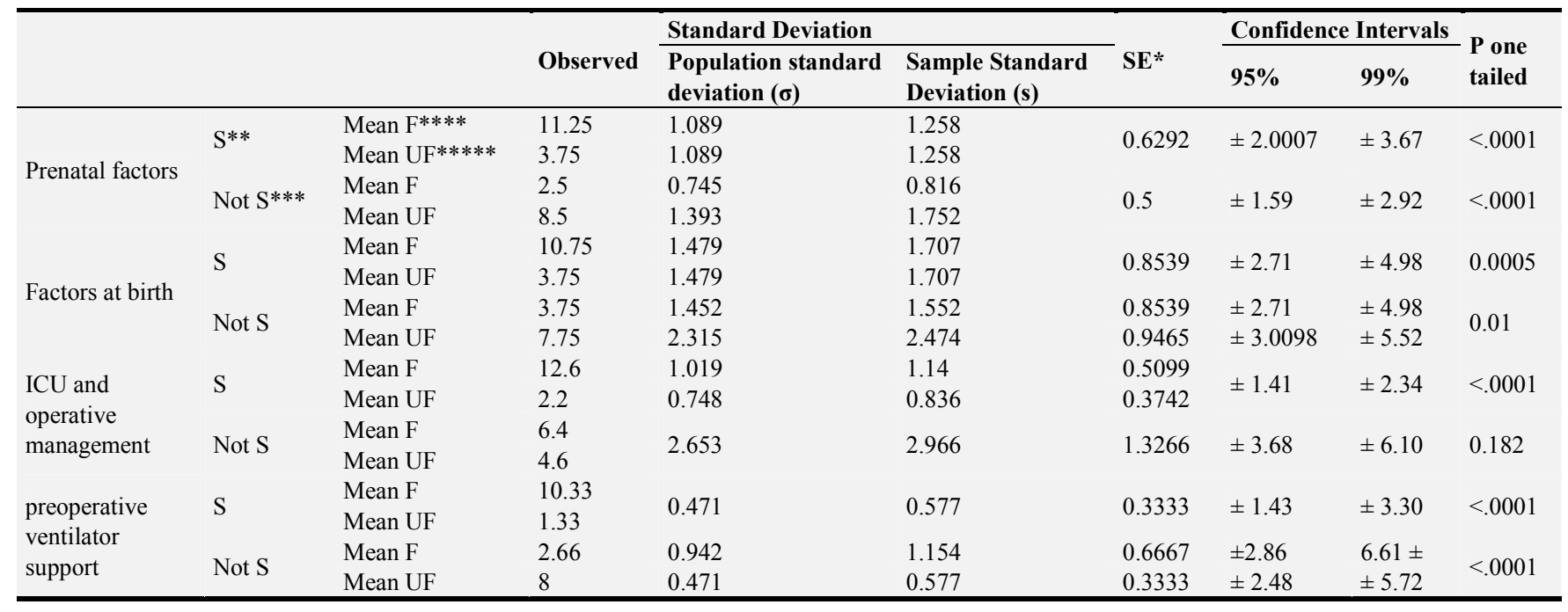

* Standard error, $* *$ survived, $* * *$ not survived, $* * * *$ favorable factors, $* * * * *$ unfavorable factors. 
Table 3. Correlation of the favorable versus unfavorable factors on the total mean outcome.

\begin{tabular}{|c|c|c|c|c|c|c|c|c|}
\hline & & \multirow[b]{2}{*}{ Observed } & \multicolumn{2}{|l|}{ Standard Deviation } & \multirow[b]{2}{*}{ SE } & \multicolumn{2}{|c|}{ Confidence Intervals } & \multirow[b]{2}{*}{$\begin{array}{l}\text { P one } \\
\text { tailed }\end{array}$} \\
\hline & & & $\begin{array}{l}\text { Population standard } \\
\text { deviation }(\sigma)\end{array}$ & $\begin{array}{l}\text { Sample Standard } \\
\text { Deviation (s) }\end{array}$ & & $95 \%$ & $99 \%$ & \\
\hline \multirow{2}{*}{ Proposed favorable outcome } & Mean S & 11.61 & 1.443 & 1.502 & 0.4166 & \pm 0.908 & \multirow{2}{*}{ \pm 1.27} & \multirow{2}{*}{$<.0001$} \\
\hline & Mean Not S & 4.53 & 2.437 & 2.536 & 0.6937 & \pm 1.533 & & \\
\hline \multirow{2}{*}{ Proposed unfavorable outcome } & Mean S & 3.23 & 1.367 & 1.423 & 0.3947 & \pm 0.908 & \pm 1.20 & \multirow{2}{*}{0.00016} \\
\hline & Mean Not S & 6.61 & 2.527 & 2.631 & 0.7298 & \pm 1.590 & \pm 2.22 & \\
\hline
\end{tabular}

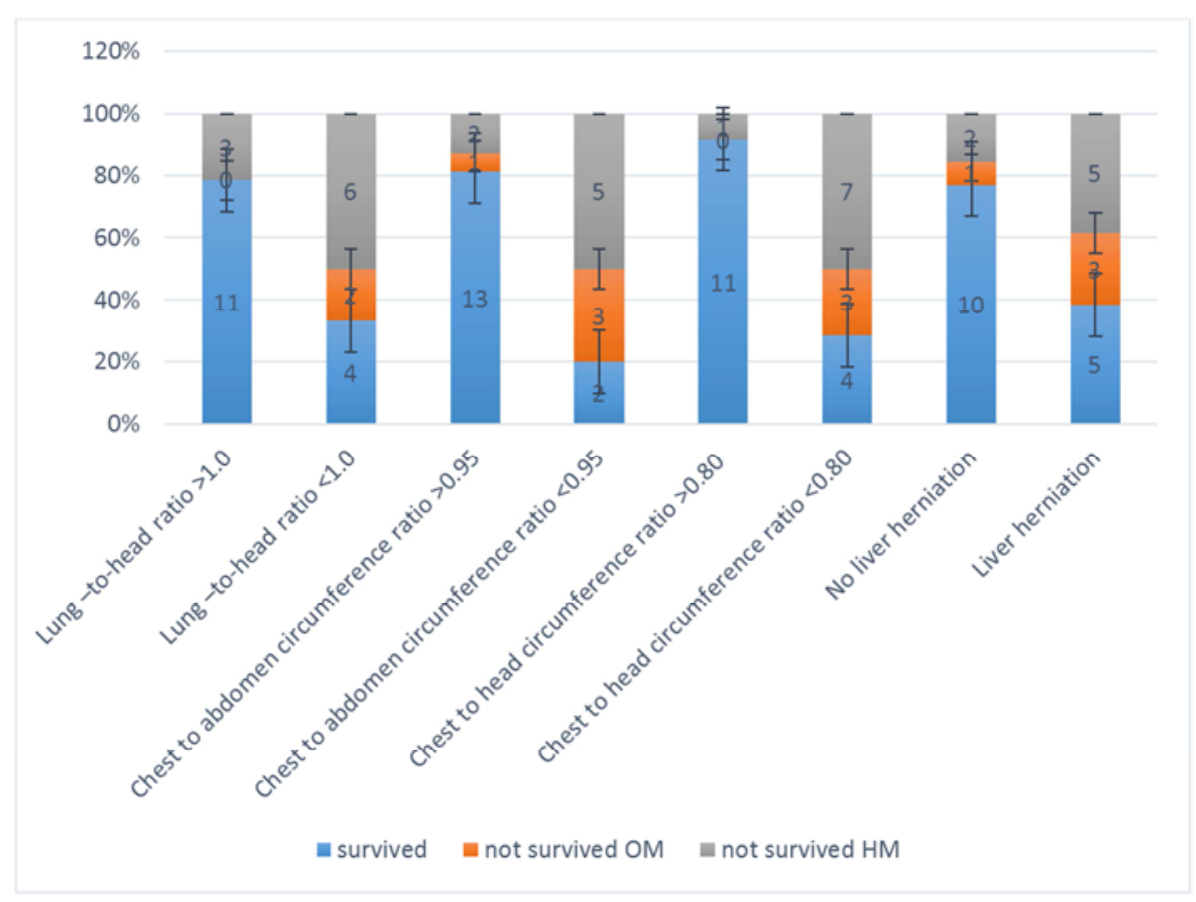

SE: 2.07 .

Figure 1. Prenatal favorable versus unfavorable factors outcome in 26 neonates out of 31 patients.

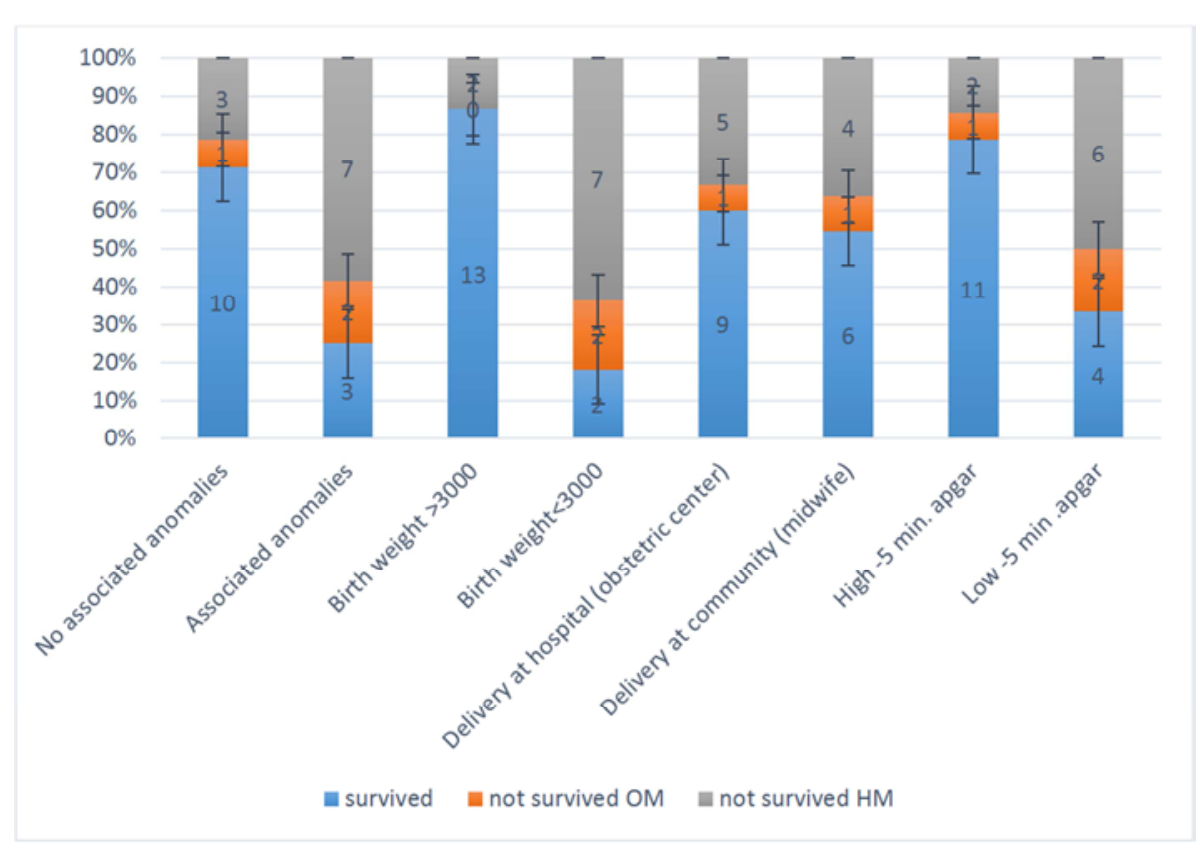

SE: 1.95 .

Figure 2. At birth level, favorable versus unfavorable factors outcome in 26 neonates. 


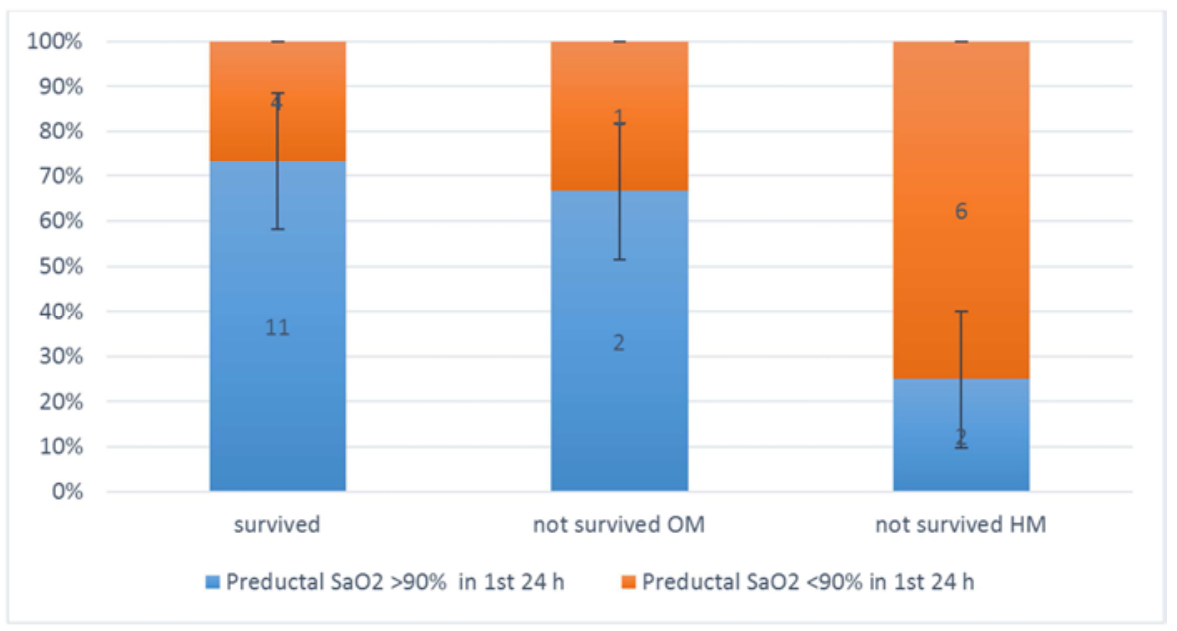

Figure 3. The detection of first 24-hour preductal $\mathrm{SaO}_{2}$ saturation outcome.

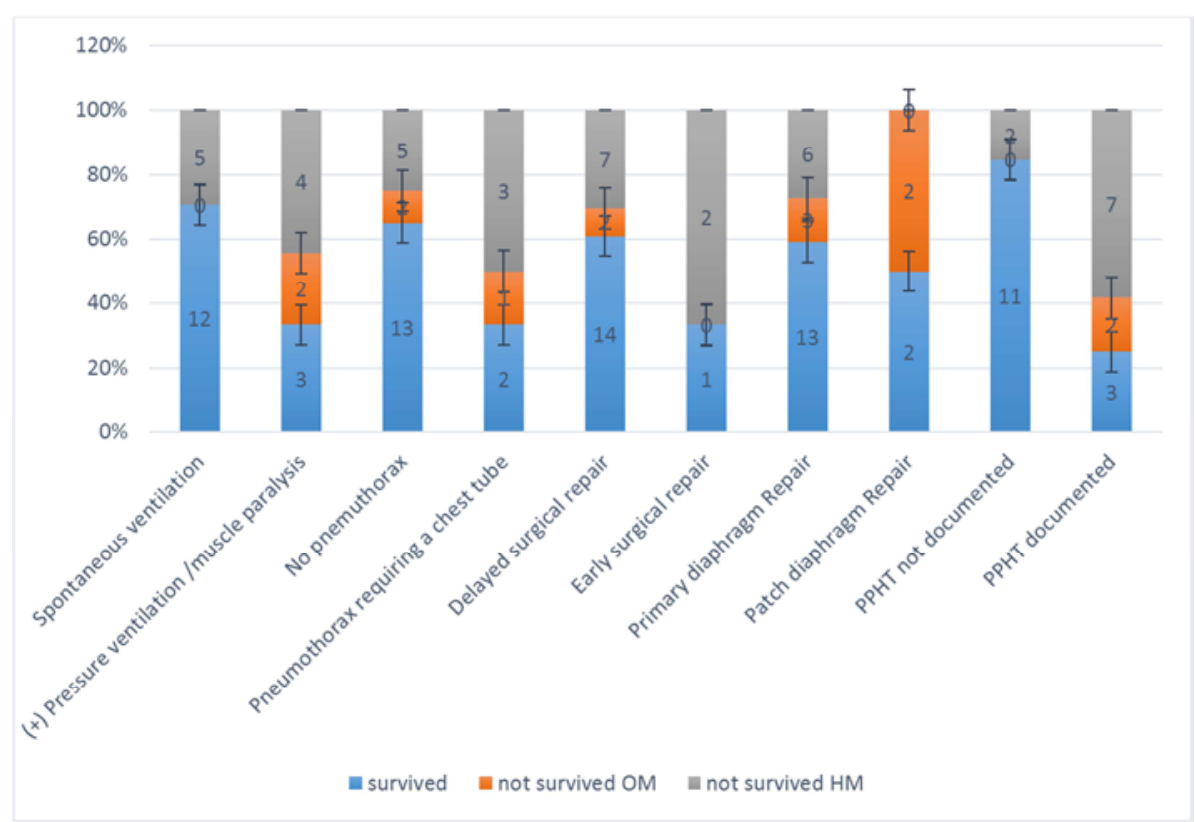

SE: 2.72 .

Figure 4. Favorable versus unfavorable factors outcome in 26 neonates at intensive care unit and operative management level.

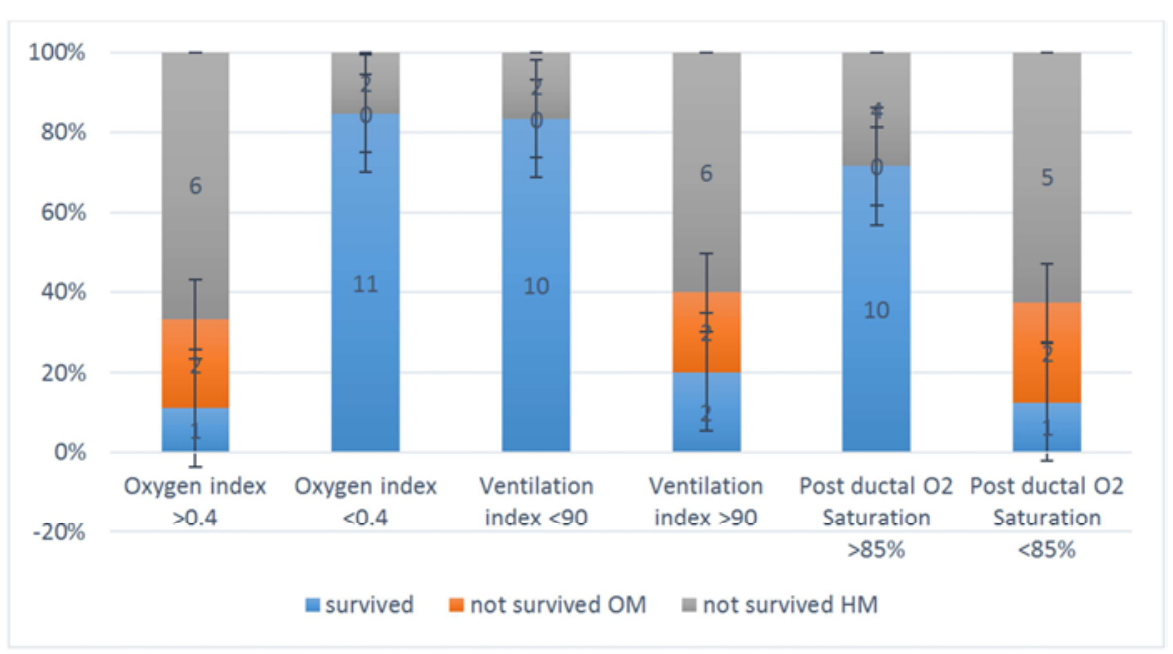

SE: 1.49 .

Figure 5. Indexes of favorable versus unfavorable factors outcome for 22 of 31 patients received preoperative ventilator support. 


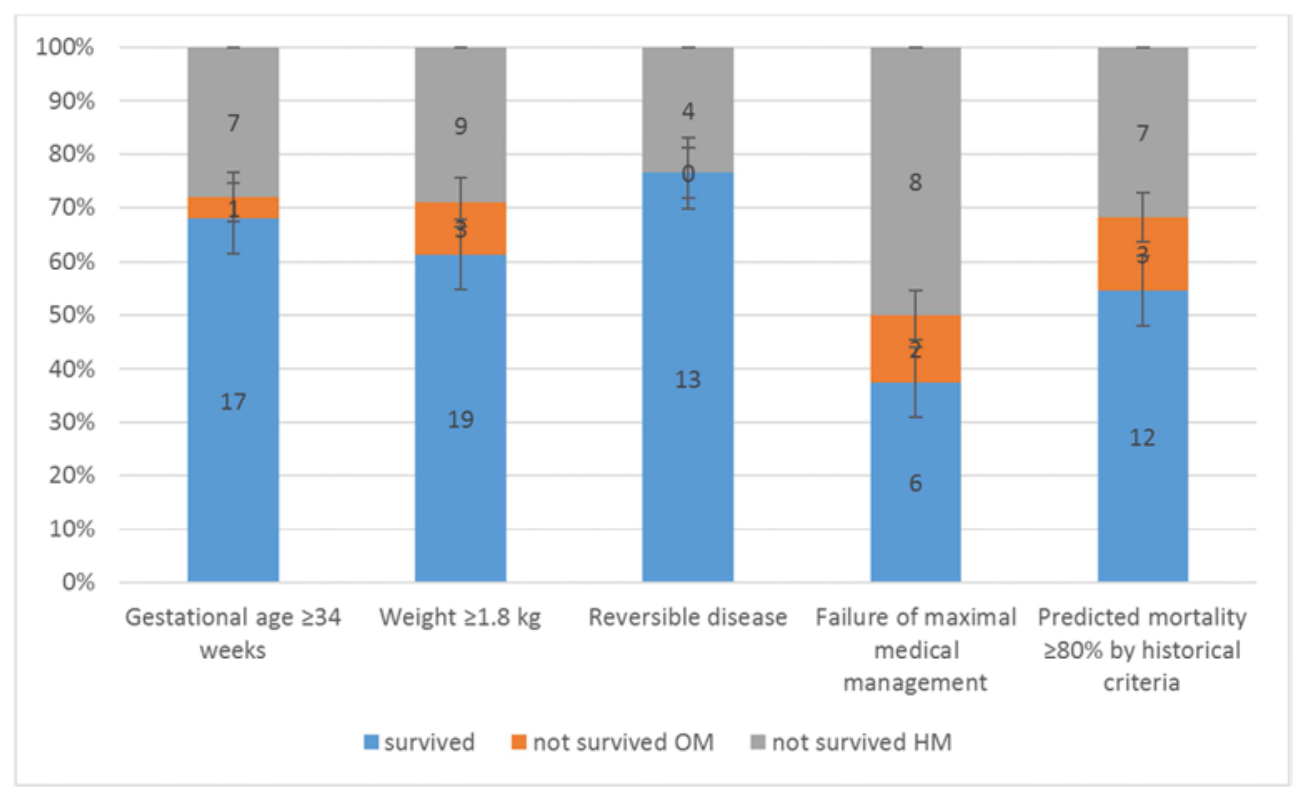

SE: 4.5

Figure 6. Indexes outcome of Patients suspected to have ECMO facilities and permissive hypercapnia according ECMO protocol.

\section{Discussion}

The optimal management of $\mathrm{CDH}$ remains unsolved. Different patients have different degrees of pulmonary hypoplasia and pulmonary hypertension secondary to the right to- left pulmonary shunting. Geggel, et al. showed hypoplastic lung not only in the ipsilateral but also in the contralateral lung of infants born with $\mathrm{CDH}$ [7].

It is well known that some live-born infants with $\mathrm{CDH}$ die before referral to a tertiary center. Consequently, the population of infants reaching a tertiary care center commonly represents only $40-50 \%$ of the total number of cases of $\mathrm{CDH}$. This disparity reflects the hidden mortality of $\mathrm{CDH}$ as first described by Harrison et al. [8]. Due to a welldeveloped prenatal screening program in Switzerland, one study assumed that the overall mortality of $\mathrm{CDH}$ patients calculated on behalf of a population- based study would be twice as high [10].

The lung-to-head ratio [9], one of the reliable predictors to help determine postnatal survival in $\mathrm{CDH}$, first described by Metkus et al. in 1996, calculated by dividing the contralateral lung area to the fetal head circumference [11]. This ratio may also depend on the gestational age at measurement and may be less reliable in mid-gestation. Recently, the ratio is being a part or used as a prenatal predictor of outcome; a measurement below $25 \%$ was reported to be associated with a less than $20 \%$ chance of survival [12]. We agreed that routine prenatal ultrasonography was not performed on all pregnant mothers, in some instance the rate of outborn delivery without a prenatal diagnosis was very high at our state this may form a limitation in our study. For other patients postnatally diagnosed we measured the expected and observed lung to head ratio to overcome this bias and to have the equal reading of this predictive parameter. Other studies reported $47 \%$ of patients with $\mathrm{CDH}$ had prenatal scans $[13$, 14]. The ratio of LHRs of $<1.0$ and $>1.4$ are strong predictors of outcome, with reported mortalities of $100 \%$ and $100 \%$ survival respectively $[15,16]$. Prenatal predictive outcome confronts parents and doctors with difficult decisions on treatment strategies, it is not yet clear which is the most reliable prenatal predictor of survival.

The incidence of associated congenital anomalies in infants with $\mathrm{CDH}$ ranges from rare to as high as $56 \%$ [17]. Cardiac anomalies are the most common, followed by genitourinary, gastrointestinal, central nervous system, skeletal and chromosomal abnormalities. The percentage of patients with coexisting major anomalies in our study population nearly was similar. This is consistent with other population-based studies, which reported rates of $37 \%$ to $47 \%[1,18]$. Multidisciplinary follow-up reported approximately $87 \%$ of $\mathrm{CDH}$ survivors have longer lasting associated morbidity, such as pulmonary, gastrointestinal and neurological problems. The presence of an additional major anomaly was associated with poor overall survival rates. Most other studies but not all found associated major anomalies to be important predictors of mortality rates [19].

Several studies mentioned the usual predictors to evaluate the patients with $\mathrm{CDH}$, used for initial clinical characteristics related to the survival rate, i.e., sex, gestational age, birth weight, Apgar score at $5 \mathrm{~min}$, associated anomalies, prenatal diagnosis, inborn delivery, polyhydramnios, and liver herniation. Among the above initial clinical characteristics, birth weight and Apgar score at 5 min were found to be the most important predictors to estimate the severity of $\mathrm{CDH}$ in the first $5 \mathrm{~min}$ of life by the Congenital Diaphragmatic Hernia Study Group [7, 8, 20].

Ventilatory support, pulmonary abnormality, and asymmetry of the chest cavity seen in $\mathrm{CDH}$ make ventilation a major challenge. Conventional mechanical ventilation 
(CMV) was the primary mode of ventilation (time-cycled, pressure limited). Systemic oxygenation status versus dynamic compliance of the respiratory system considered Predictors of survival in infants with congenital diaphragmatic hernia [21]. In our series, the patients candidate for ventilator support (received CMV) were paralyzed and received a continuous infusion of fentanyl (5 $10 \mu \mathrm{g} / \mathrm{kg} / \mathrm{h})$ and dopamine $(2.5 \mu \mathrm{g} / \mathrm{kg} / \mathrm{min})$. May this approach carried some risk as many studies reported that the paralysis better to be avoided, as it may have negatively adverse effects on ventilation with prolonged mechanical ventilation and oxygen toxicity, all can cause lung damage and predict the mortality $[22,23]$.

Gentle ventilation with adequate oxygenation defined as preductal oxygen saturations of greater than $85 \%$, allowing minimization of peak inspiratory pressure to less than $20 \mathrm{~cm}$ $\mathrm{H}_{2} \mathrm{O}$. This represents the most significant advancement in CDH management, with marked improvements in survival, from $50 \%$ to $89 \%$ and reduction in complications of barotrauma, survival rates of more than $80 \%$ in patients with isolated $\mathrm{CDH}$ have been achieved in several centers using gentle ventilation [24].

Ventilation index (VI) used to determine the severity of respiratory in critically ill patients [25]. A different study in the literature demonstrated that survival rate was $91 \%$ if VI score was under 40 in patients with CDH [26]. During the study, the VI was not different at the first hours in the patients who lived, compared with those who subsequently died. However, by 1 to 3 days after, the VI of nonsurvivors was significantly higher than for survivors. The VI for survivors remained between 35 and 45 throughout the admission period, whereas the VI of nonsurvivors continued to increase with time. A VI of $>90$ predicted death in 8 patients on days 2 through 5 . The VI provides a reliable prognostic marker in children with $\mathrm{CDH}$, and its increase above 85-90 indicates a need for orderly intervention with alternative modalities of care.

Ideally, the patients must maintain $\mathrm{O}_{2}$ saturations $>95 \%$ with gentle ventilation and vascular support limits. If this cannot be achieved, resources of ECMO may be enrolled as alternative support, again this facility not present during our series study. We followed such consequences by measuring OI, Our data revealed that $80 \%$ the $\mathrm{CDH}$ patients received preoperative ventilator support with OI $>90 \%$ died. Other researchers reported, the higher OI predicts a high mortality and has been widely used as criteria for ECMO [27]. Studies using multiple logistic regression analysis identified two adverse prognostic factors: an Apgar score at 1 min (Ap1) of $0-4$, and a best oxygenation index (OI) 8.0, low Apgar scores and high oxygenation index (OI) were associated with poor outcome in infants with $\mathrm{CDH}$ (higher OI also reflects and triggering the more severe PPHN in comparison to survivors [28].

Comparisons of survival rates of $\mathrm{CDH}$ patients between institutions or periods are difficult and controversial because the spectrum of disease severity is very wide. Recently, the Congenital Diaphragmatic Hernia Study Group reported an overall survival rate of $64 \%$ based on data from institutions in North America, Europe, and Australia [5, 7, 11, 12]. Thus, the overall survival rate of at our institution (a 9 years study) is nearly similar to those reported from other institutions regardless the applied resources, however, the survival rate at our institution has not been constant over this period. Our study, in a little bit the results underscores the improved survival rate for patients with $\mathrm{CDH}$ with all the challenges mentioned.

An important factor when comparing surgical results (and especially survival) in different studies is the ratio early (neonates) vs. late presenters. The children that present late usually have smaller hernias that do not cause any major respiratory problems at birth $[29,30]$. These children do not need intensive care and chest X-ray not performed after birth, this leads to diagnostic delay, often for months. In our series, five children were late presenters. All of these patients survived surgery without major postoperative complications or need for ventilator treatment, underscoring the good prognosis of this subgroup.

In a recent prospective study, there was neither an advantage to urgent surgical repair (we practiced that in 3 patients) nor a clear advantage to a long delay for repair in clinically stable infants [31]. Our current approach to the treatment of infants with $\mathrm{CDH}$ is to attain medical stabilization before subjecting these infants to surgical repair (now this is the role of our unit). The impact of timing of surgery on survival is a debated issue for patients with $\mathrm{CDH}$ $[32,33]$.

We agreed that there is a significant limitation due to the small number of patients who included in our study and other some limitations to adjust for all initial clinical characteristics due to its retrospective nature. Further prospective studies will be required to overcome these limitations and to confirm our hypothesis raised in this study.

In conclusion, Infants with $\mathrm{CDH}$ had a varying degree of compromise that is difficult to evaluate before birth, therefore, proved patients should be born at a qualified pediatric tertiary center with a multimodality support available, unfortunately, important facilities still not present in our unit. A composite of prognostic and predictive parameters, however, organize our workup and can magnify our management future policy despite that, these prognostic indicators measure different aspects of $\mathrm{CDH}$.

\section{Acknowledgments}

The author expresses sincere gratitude to all the pediatric surgery unit staff at the Maternity and Child Teaching Hospital, Al-Qadisiya, Iraq, for their assistance and efforts to obtain the results and all colleagues in radiology and pediatric units. Thanks also go to professor Manal Mohammed (head of CME unit, college of Medicine, Al Qadisiya University) for her kind revision to the statistical analysis. 


\section{References}

[1] Fitzgerald RJ. Congenital diaphragmatic hernia: a study of mortality factors. Irish J Med Sci 1997; 146: 280-4.

[2] Bohn D, Tamura M, Perrin D, Barker G, Rabinovitch M. Ventilatory predictors of pulmonary hypoplasia in congenital diaphragmatic hernia confirmed by morphologic assessment. J Pediatr 1987; 3: 423- 31.

[3] Sakai H, Tamura M, Hasokama Y, Bryan AC, Barber GA, Bohn D. Effect of surgical repair in respiratory mechanics in congenital diaphragmatic hernia. J Pediatr 1987; 3: 432-8.

[4] Hussein Khawahur, MD; Abdulhakiem Kattan, MD; Congenital diaphragmatic hernia: a local experience. Annals of Saudi Medicine, Vol 19, No 6, 1999.

[5] Clark RH, Hardin WD Jr, Hirschl RB, Jaksic T, Lally KP, Langham MR Jr, Wilson JM. Current surgical management of congenital diaphragmatic hernia: a report from the congenital diaphragmatic hernia study group. J Pediatr Surg 1998; 33: 1004-9.

[6] Beresford MW, Shaw NJ. Outcome of congenital diaphragmatic hernia. Pediatr Pulmonol 2000; 30: 249-256.

[7] The Congenital Diaphragmatic Hernia Study Group. Estimating disease severity of congenital diaphragmatic hernia in the first 5 minutes of life. J Pediatr Surg 2001; 36: 141-5.

[8] Do-Hyun Kim, June Dong Park, Han-Suk Kim, So-Yeon Shim, Survival Rate Changes in Neonates with Congenital Diaphragmatic Hernia and its Contributing Factors, J Korean Med Sci 2007; 22: 687-92.

[9] Jason C. Fisher *, Rashida A. Jefferson, Marc S. Arkovitz, Charles J. H. Stolar, Redefining outcomes in right congenital diaphragmatic hernia, Journal of Pediatric Surgery (2008) 43, 373-379.

[10] Thibeault DW, Haney B. Lung volume, pulmonary vasculature, and factors affecting survival in congenital diaphragmatic hernia. J Pediatr Surg 1998; 101: 289-295.

[11] Macken MB, Thompson DL. Antenatal diagnosis of congenital diaphragmatic hernia. Can Assoc Radiol J 1993; 44: 439-42.

[12] Midrio P, Gobbi D, Baldo V, et al. Right congenital diaphragmatic hernia: an 18-year experience. J Pediatr Surg 2007; 42: 517-21.

[13] SteinhornRH, KriesmerPJ, GreenTP, McKayCJ, PayneNR. Congenital diaphragmatic hernia in Minnesota: impact of antenatal diagnosis on survival. Arch Pediatr Adolesc Med. 1994; 148: 626-631.

[14] Lewis DA, Reickert C, Bowerman R, Hirschl RB. Prenatal ultrasonography frequently fails to diagnose congenital diaphragmatic hernia. J Pediatr Surg. 1997; 32: 352-356.

[15] Laudy JA, Van Gucht M, Van Dooren MF, Wladimiroff JW, Tibboel D. Congenital diaphragmatic hernia: an evaluation of the prognostic value of the lung-to-head ratio and other prenatal parameters. Prenat Diagn 2003; 23: 634-639.

[16] Lipshutz GS, Albanese CT, Feldstein VA et al. Prospective analysis of lung-to-head ratio predicts survival for patients with prenatally diagnosed congenital diaphragmatic hernia. J
Pediatr Surg 1997; 32: 1634-1636.

[17] Nio M, Haase G, Kennaugh J, Bui K, Atkinson JB. A prospective randomized trial of delayed versus immediate repair of congenital diaphragmatic hernia. J Pediatr Surg 1994; 29: 618-21.

[18] Norden MA, Butt W, McDougall P. Predictors of survival for infants with CDH. J Pediatr Surg 1994; 29: 1442-6.

[19] Joanne Colvin, Carol Bower, Jan E. Dickinson and Jenni Sokol, Outcomes of Congenital Diaphragmatic Hernia: A Population-Based Study in Western Australia, Pediatrics: DOI: 10.1542/peds.2004-2845 2005; 116; e356-e363.

[20] Skari H, bjornland K, Frenckner b, Friberg LG, Heikkinen M, Hurme T, Loe b, Mollerlokken G, Nielsen OH, Qvist N, Rintala $\mathrm{R}$, Sandgren $\mathrm{K}$, Wester $\mathrm{T}$, Emblem R: Congenital diaphragmatic hernia in Scandinavia from 1995 to 1998: Predictors of mortality. J Pediatr Surg 2002; 37 (9): 1269-1275.

[21] Lieke van den Hout, Ilona Sluiter, Saskia Gischler, Annelies de Klein, Robert Rottier, Hanneke IJsselstijn, Irwin Reiss, Dick Tibboel, Can we improve outcome of congenital diaphragmatic hernia? Pediatr Surg Int, 2009; 25 (9): p. 733743.

[22] Bancalari E, Claure N. Definitions and diagnostic criteria for bronchopulmonary dysplasia. Semin Perinatol. Aug 2006; 30 (4): 164-170.

[23] Bancalari E, Claure N, Sosenko IR. Bronchopulmonary dysplasia: changes in pathogenesis, epidemiology and definition. Semin Neonatol. Feb 2003; 8 (1): 63-71.

[24] Paul D. Robinson and Dominic A. Fitzgerald, Congenital diaphragmatic hernia, pediatric respiratory review (2007) 8, 323-335.

[25] Paret G, Ziv T, Barzilai A, BenAbraham R, Vardi A, Manisterski Y, Barzilay Z. Ventilation index and outcome in children with acute respiratory distress syndrome. Pediatr Pulmonol. 1998 Aug; 26 (2): 1258.

[26] Z lçe, C Güney, N Eray, B Ilikkan, S Celayir. The Role of Modified Ventilatory Index in Defining the Prognosis in Surgical and Nonsurgical Pediatric Patients. The Internet Journal of Pulmonary Medicine. 2004 Volume 5 Number 1.

[27] Luis R. Longo dos Santos, João G. Maksoud-Filho, Uenis Tannuri, Wagner C. Andrade, João G. Maksoud. Prognostic factors and survival in neonates with congenital diaphragmatic hernia. J Pediatr (Rio J) 2003; 79 (1): 81-6.

[28] Ruano R, Bunduki V, Silva MM, Yoshizaki CT, Tanuri U, Macksoud JG, et al. Prenatal diagnosis and perinatal outcome of 38 cases with congenital diaphragmatic hernia: 8-year experience of a tertiary Brazilian center. Clinics 2006; 61: 197-202.

[29] Smith NP, Jesudason EC, Featherstone NC, Corbett HJ, Losty PD: Recent advances in congenital diaphragmatic hernia. Arch Dis Child 2005; 90 (4): 426-428.

[30] 28. Baglaj M: Late-presenting congenital diaphragmatic hernia in children: a clinical spectrum. Pediatr Surg Int 2004; 20 (9): 658-669.

[31] Wilson JM, Fauza DO, Lund DP, Benacerraf BR, Hendren WH. Antenatal diagnosis of isolated congenital diaphragmatic hernia is not an indicator of outcome. J Pediatr Surg 1994; 29: 815-9. 
[32] Langer JC, Filler RM, bohn DJ, Shandling b, Ein SH, Wesson DE, Superina RA: Timing of surgery for congenital diaphragmatic hernia: is emergency operation necessary? J Pediatr Surg 1988; 23 (8): 731-734.
[33] Smith NP, Jesudason EC, Featherstone NC, Corbett HJ, Losty PD: Recent advances in congenital diaphragmatic hernia. Arch Dis Child 2005; 90 (4): 426-428. 